\title{
Up-converted luminescence of cyanine dye J-aggregates
}

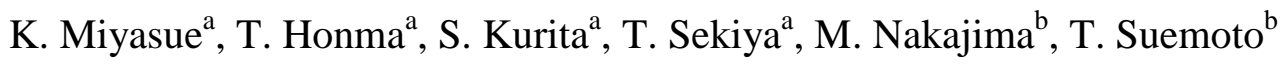 \\ ${ }^{a}$ Department of Physics, Faculty of Engineering, Yokohama National University \\ Tokiwadai 79-5, Hodogaya, Yokohama 240-8501, Japan \\ ${ }^{\mathrm{b}}$ Institute for Solid State Physics, The University of Tokyo \\ Kashiwanoha, 5-1-5, Kashiwa-shi, Chiba 277-8581, Japan
}

[received :

\begin{abstract}
A J-band photoluminescence of cyanine dye J-aggregates was observed under excitation below the band gap. This emission is not due to two-photon absorption. The luminescence intensity becomes weaker with a decrease in temperature. For the excitation below the band gap, the rise and decay times of the J-band luminescence from cyanine dye concentration of $4 \times 10^{-4} \mathrm{M}$ are $9.0 \mathrm{ps}$ and $155 \mathrm{ps}$, respectively. In case of the band-to-band excitation, the radiative decay starts just after excitation and fast and slow decay components have time constants of 13 and 54 ps, respectively. The lifetime of luminescence depends on the concentration of the cyanine dye.
\end{abstract}

\section{Keywords}

J-aggregate, J-band, Time-resolved luminescence, Cyanine dye,

\section{Pacs code}

36.40.Vz; 78.55.Kz; 78.47.+p

\section{Corresponding Author}

Takao SEKIYA

Department of Physics, Faculty of Engineering,

Yokohama National University,

Tokiwadai 79-5, Hodogaya, Yokohama, 240-8501, Japan

Tel: +81-45-339-3954

Fax: +81-45-339-3954

e-mail sekiya@ynu.ac.jp (T. Sekiya) 


\section{Introduction}

J-aggregates of cyanine dye being long chains of identical molecules arranged in one dimensional structure are characterized by a sharp J-band red-shifted from the monomer band [1]. The J-band is explained as due to a Frenkel exciton moving along the chain [2-4]. Recently, we reported that a J-band luminescence of cyanine dye J-aggregates can be seen under the excitation below the band gap, that is, J-band luminescence at $2.08 \mathrm{eV}$ was observed by He-Ne laser $(1.96 \mathrm{eV})$ excitation. This phenomenon is not due to two-photon absorption [5]. The spectrum of the J-band luminescence excited by a He-Ne laser resembles that observed under band-to-band excitation due to an $\mathrm{Ar}^{+}$laser $(2.41 \mathrm{eV})$. This involves that their final excitonic states are identical. In this report, the temperature dependence of the luminescence and the effect of the dye concentration were investigated.

\section{Experimental}

The cyanine dye 5, 6-Dichloro-2-[3-[5, 6-dichloro-1-ethyl-3-(3-sulfopropyl)-2(3H)benzimidazolylidene]-1-propenyl]-1-ethyl-3-(3-sulfopropyl)benzimidazolium hydroxide, inner salt, sodium salt (NK-2203, Japanese Research Institute for Photo-sensitizing Dyes Co.) was used without further purification. In this experiment, samples of aqueous solutions with various concentrations and of spin-coated film were used. In aqueous solution, the J-band absorption is located at $2.09 \mathrm{eV}$ and the two absorption bands observed at 2.42 and $2.58 \mathrm{eV}$ are said to be due to the monomer and the dimer, respectively [6]. The spin-coated film was made by the method proposed by Misawa and Kobayashi [7]. The dye dispersed in polyvinyl alcohol with distilled water was spin-coated on a glass substrate. The absorption due to the monomer or to the dimer was not observed for the film sample. The photoluminescence of J-aggregate was measured in the same manner previously used [5]. The spectra of the J-band luminescence are quite similar for both samples, the aqueous solution and the spin-coated film.

\section{Results and discussion}

In order to estimate the change in absorption intensity at the tails of J-band absorption, we measured the temperature dependence of the optical absorption of the spin-coated film. The result is shown in Fig. 1. The peak energy of the absorption band is $2.09 \mathrm{eV}$ at room temperature. The peak shifts slightly to the higher energy side. The absorption tails on the high and low energy sides of the J-band have little change with temperature. Therefore, we assume no change of the absorption intensity at the two excitation energies 2.41 and $1.96 \mathrm{eV}$ with temperature.

Temperature dependence of $\mathrm{J}$-band luminescence corresponding to the band-to-band excitation using $\mathrm{Ar}^{+}$laser $(2.41 \mathrm{eV})$ is shown in Fig. 2(a). The peak energy of the luminescence band is $2.09 \mathrm{eV}$ at room temperature. The luminescence band shows slight blue-shift and narrowing with a decrease in temperature. The intensity of the luminescence increases with a decrease in temperature, shown in Fig. 3. Generally, the luminescence 
intensity is proportional to the quantum efficiency, $\eta=\frac{A}{A+s \exp (-\Delta W / k T)}$, where $A, s$ and $\Delta W$ are the transition probability of luminescence, the frequency factor and the activation energy, respectively. The result of the fitting is also shown by the solid line in Fig. 3. The activation energy is estimated to be about $24 \mathrm{meV}$. This suggests that the probability of non-radiative decay process is relatively large at room temperature. These facts are quite reasonable.

Figure 2(b) shows the luminescence obtained by the excitation on the low energy side. The irradiation with He-Ne laser light $(1.96 \mathrm{eV})$ at room temperature results in the observation of the J-band luminescence located at $2.08 \mathrm{eV}$. It is worth to notice that, in spite of the excitation below the band gap, the J-band luminescence can be observed at a higher energy side, about $0.12 \mathrm{eV}$ above the excitation energy. The direct proportion of the luminescence intensity to the excitation intensity clearly indicated that the luminescence was not due to two-photon absorption [5]. The energy difference is much larger than the thermal energy available at room temperature $(26 \mathrm{meV})$. The decrease in temperature results in a slight blue-shift of the peak of the luminescence band and a small narrowing of the band width. The luminescence band becomes suddenly weak with a decrease in temperature, as shown in Fig. 3. This temperature dependence of the luminescence intensity can be fitted using an Arrhenius-type function, $L=L_{0} \exp \left(-\frac{\Delta E}{k T}\right)$. As a result, we obtain the activation energy $\Delta E=0.13 \mathrm{eV}$. This value is compared to the energy difference between the peak of the J-band luminescence and the excitation energy provided by the He-Ne laser. This suggests that some thermal effect participates in the up-converted luminescence, in spite the fact that the thermal energy at room temperature seems being too small to excite the J-band.

Time-resolved luminescence due to the band-to-band excitation of the aqueous solution was measured at $2.08 \mathrm{eV}$ for an excitation at $2.24 \mathrm{eV}$ at room temperature, as shown in Fig. 4. The luminescence was observed just after the excitation. The decay curve seems to be well described by two components of simple exponential functions. The decay curves calculated by the convolution of simple exponential function with the system response are also plotted in Fig. 4. The lifetimes estimated by the curve fitting are listed in Table 1. As the dye concentration of the solution increases, each lifetime of the two components becomes shorter. It is noted that the film sample gives the decay curve represented by a single exponential function, as reported previously [5].

Figure 5 shows time evolution of the luminescence measured at $2.08 \mathrm{eV}$ for an excitation at $2.00 \mathrm{eV}$. The luminescence due to the excitation below the band gap rises slowly in contrast to the band-to-band excitation. This indicates that the time evolution of the luminescence can be represented by the function $I(t)=I_{0}\left(1-e^{-t / \tau_{r}}\right) e^{-t / \tau_{d}}$. This function was convoluted with the line shape of the laser pulse to agree with the observed time evolution. The obtained parameters are listed in Table 1 and the fitting curve is drawn in Fig. 5. The rise and decay 
times, $\tau_{\mathrm{r}}$ and $\tau_{\mathrm{d}}$, of the solution with high concentration are shorter than those with low concentration. This change of the time parameters with the concentration suggests the presence of an interaction between two adjacent J-aggregates. We propose one possible model of this up-converted luminescence due to the excitation below the band gap as follows: J-aggregate excited below the band gap absorbs the insufficient energy as phonons to be excited to the J-band, and relax to ground state through the radiative path. The slow rise time and long decay time for J-band luminescence will be due to the small transition probability of the phonon absorption and to the drop in temperature of the J-aggregate, respectively. One may consider the Förster transfer as the energy transfer mechanism between photoexcited and unexcited J-aggregates.

\section{Summary}

The temperature dependence of the luminescence of a J-aggregate film was measured for the band-to-band excitation as well as for an excitation below the band gap. The luminescence due to the low energy side excitation becomes suddenly weak with a decrease in temperature. The luminescence intensity shows Arrhenius-type change depending on temperature. The time-resolved luminescence was measured at room temperature using aqueous solutions with different cyanine dye concentration. When an aqueous solution of $4.0 \times 10^{-4} \mathrm{M}$ is excited by an energy higher than the J-band, the presence of two decay components with life time of 10 and $51 \mathrm{ps}$ is elucidated. On the excitation at a lower energy than the J-band, the luminescence rises up slowly. Curve fitting reveals that the rise and decay time constants are 9.0 and $155 \mathrm{ps,}$ respectively. These time parameters have temperature dependence. Our possible model for the up-converted luminescence observed under excitation at the low energy side is that $\mathrm{J}$-aggregate excited below the band gap receives some phonons to be excited to the J-band.

\section{References}

[1] G. Scheibe, Angrew. Chem. 49 (1936) 563.

[2] E. W. Knapp, Chem. Phys. 85 (1984) 73.

[3] P. O. J. Scherer, S. F. Fischer, Chem. Phys. 86 (1984) 269.

[4] J. Knoster, J. Chem. Phys. 99 (1993) 8466.

[5] S. Kurita, T. Honma, H. Nakamura, T. Sekiya, M. Nakajima and T. Suemoto, J. Lumin. 108 (2004) 15.

[6] B. Kopainsky, J. K. Hellermeir, W. Kaiser, Chem. Phys. Lett. 83 (1981) 498.

[7] K. Misawa, H. Ono, K. Minoshima, and T. Kobayashi, Appl. Phys. Lett. 63 (1993) 577. 


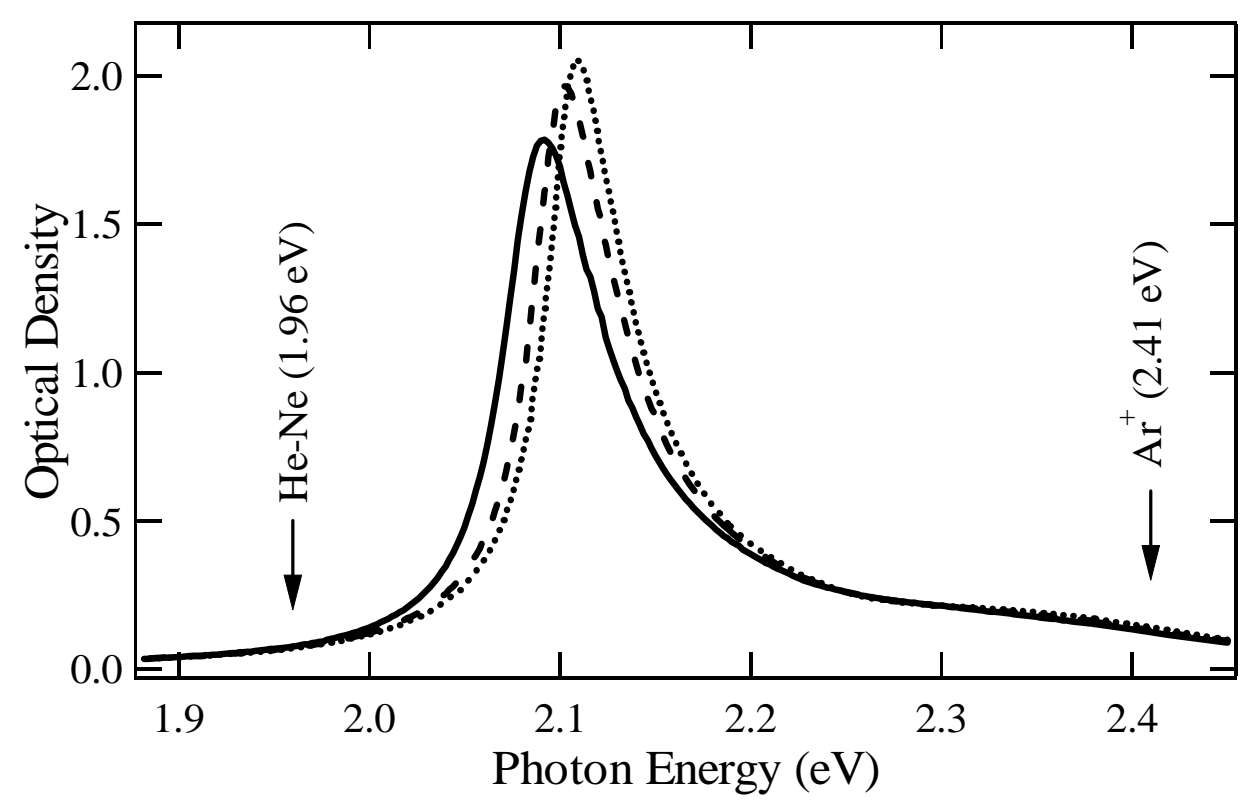

Fig. 1 Temperature dependence of optical absorption of the spin-coated film measured for non-polarization. The solid, dashed and dotted lines are spectra measured at 80, 180 and $295 \mathrm{~K}$, respectively. 

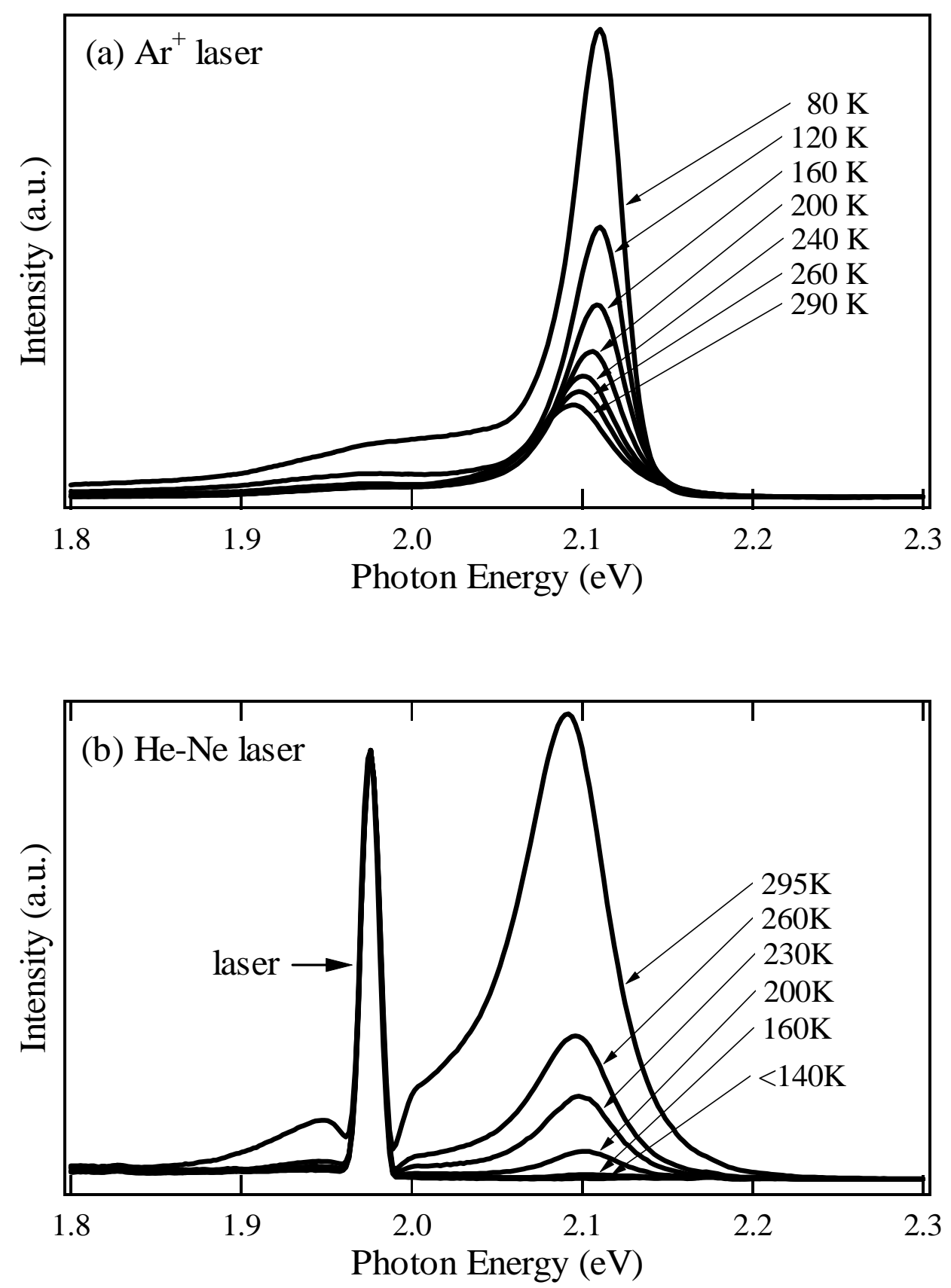

Fig. 2 Temperature dependence of luminescence spectra of the spin-coated film obtained by the irradiation of (a) $\mathrm{Ar}^{+}$and (b) He-Ne laser lights. The sharp band at $1.96 \mathrm{eV}$ is a stray light from the He-Ne laser laser. 


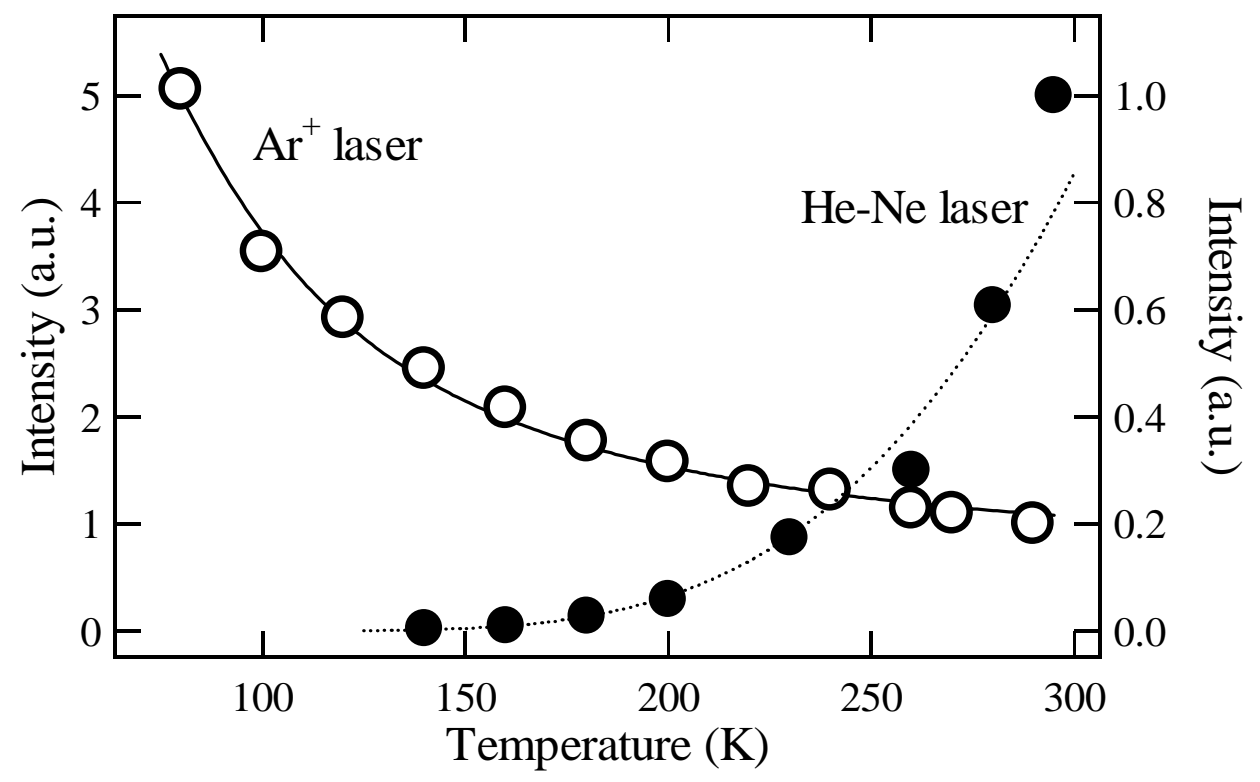

Fig. 3 Changes of luminescence intensities with temperature. The intensities were normalized to the data at $290 \mathrm{~K}$ to be 1.00 . The solid and dotted lines are analytical results based on the quantum efficiency and Arrhenius-type function, respectively. 


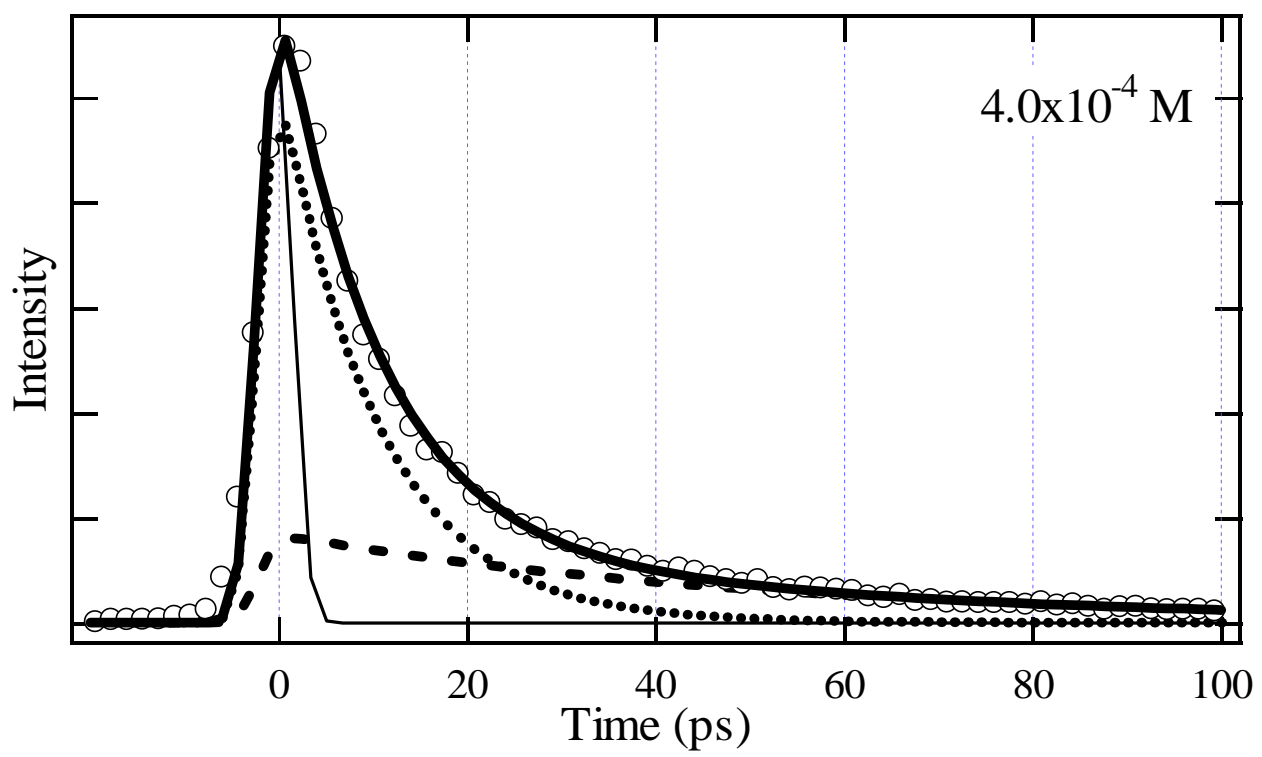

Fig. 4 Time-resolved luminescence due to band-to-band excitation on aqueous solution of $4.0 \times 10^{-4} \mathrm{M}$ at room temperature. The excitation and observation were done at 2.24 and $2.08 \mathrm{eV}$, respectively. The fast and slow components are also plotted by dashed and dotted curves, respectively. The thick and thin solid curves are the sum of them and the instrumental function (laser line), respectively.

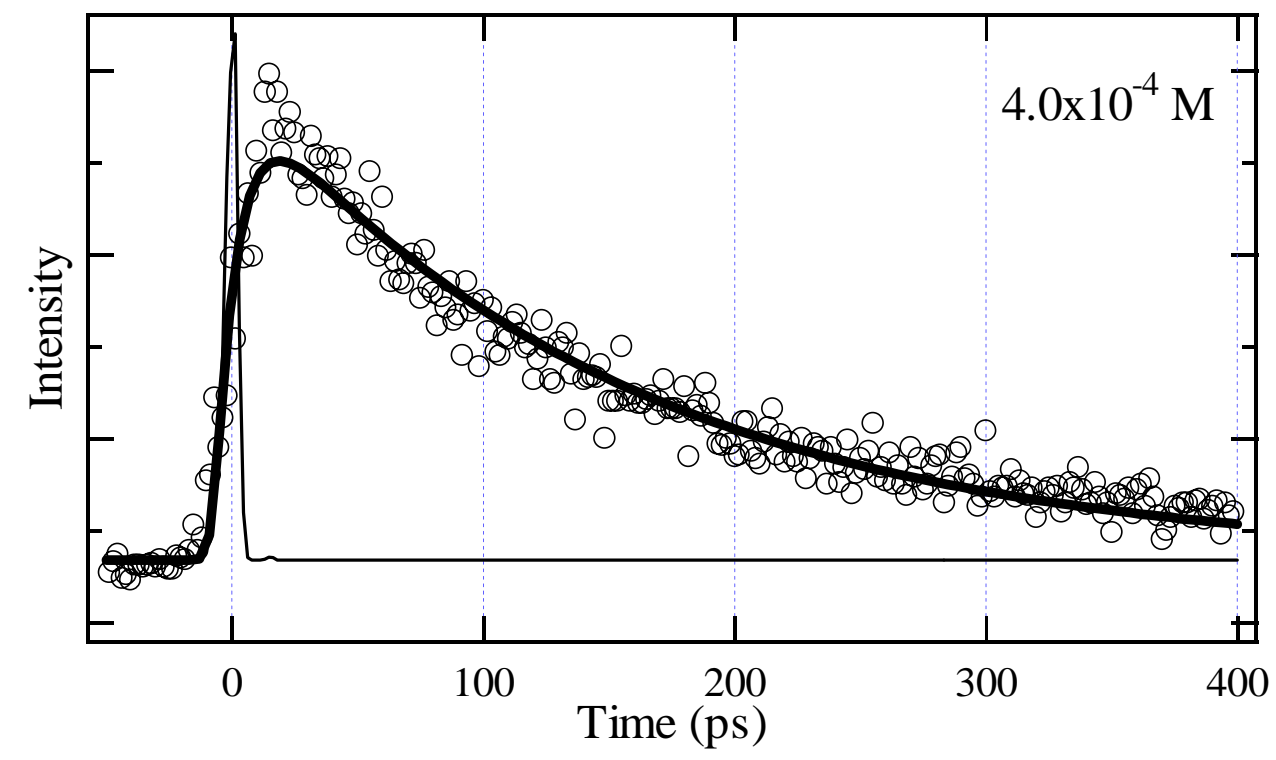

Fig. 5 Time evolution of luminescence due to the low energy side excitation on aqueous solution of $4.0 \times 10^{-4} \mathrm{M}$ at room temperature. The excitation and observation were done at 2.00 and $2.08 \mathrm{eV}$, respectively. The thick solid line represents the results of curve fitting based on the function $I(t)=I_{0}\left(1-e^{-t / \tau_{r}}\right) e^{-t / \tau_{d}}$. The thin solid curve is the instrumental function (laser line). 
Table 1 Parameters obtained by the decay curve fitting.

\begin{tabular}{llllll}
\hline \multirow{2}{*}{ Concentration } & \multicolumn{2}{l}{ band-to-band excitation } & & \multicolumn{2}{l}{ low energy side excitation } \\
\cline { 2 - 3 } \cline { 5 - 6 } \cline { 5 - 6 } & $\tau_{1}$ (decay time) & $\tau_{2}($ decay time $)$ & & $\tau_{\mathrm{r}}$ (rise time $)$ & $\tau_{\mathrm{d}}$ (decay time) \\
\hline $4.0 \times 10^{-4} \mathrm{M}$ & $10 \mathrm{ps}$ & $51 \mathrm{ps}$ & & $9.0 \mathrm{ps}$ & $155 \mathrm{ps}$ \\
$2.0 \times 10^{-4} \mathrm{M}$ & $14 \mathrm{ps}$ & $68 \mathrm{ps}$ & & $12 \mathrm{ps}$ & $155 \mathrm{ps}$ \\
$8.0 \times 10^{-5} \mathrm{M}$ & $20 \mathrm{ps}$ & $99 \mathrm{ps}$ & & $15 \mathrm{ps}$ & $195 \mathrm{ps}$ \\
\hline
\end{tabular}

